Journal of Molecular Structure, 95 (1982) 23-36

Elsevier Scientific Publishing Company, Amsterdam - Printed in The Netherlands

\title{
SPECTROSCOPIC STUDIES OF LEWIS ACID-BASE COMPLEXES
}

\author{
Part V. Raman and infrared spectra, assignments and normal coordinate \\ analysis for difluorophosphine borane
}

\author{
ROBERT C. TAYLOR and VIRGINIA D. DUNNING \\ Department of Chemistry, The University of Michigan, Ann Arbor, Michigan 48109 \\ (U.S.A.)
}

(Received 12 January 1982)

\begin{abstract}
Raman spectra of five isotopic species of $\mathrm{HPF}_{2} \mathrm{BH}_{3}$ have been obtained in the liquid and solid states together with infrared spectra of the same species in the gaseous and solid states. Vibrational assignments have been made with the aid of a normal coordinate analysis utilizing compliance constants. Both compliance and force constants are reported. The force field is quite similar to that of $\mathrm{PF}_{3} \mathrm{BH}_{3}$ despite the marked difference in stability of the two compounds.
\end{abstract}

\section{INTRODUCTION}

Difluorophosphine, $\mathrm{HPF}_{2}$, is a compound with interesting chemical properties, particularly with respect to its ability to act as a Lewis base. The related compounds, $\mathrm{PH}_{3}$ and $\mathrm{PF}_{3}$, are both extremely weak donors and their complexes with borane, $\mathrm{BH}_{3}$, are thermally unstable and nearly completely dissociated at room temperature. In contrast, $\mathrm{HPF}_{2} \mathrm{BH}_{3}$ is undissociated under the same conditions and stable indefinitely when protected from the atmosphere [1] . Satisfactory explanations for this difference in behavior are still being sought. Initial attempts to rationalize the behavior of the three complexes in terms of structural differences and the ability of the $\mathrm{BH}_{3}$ group to approach the phosphorus atom when forming the dative bond do not appear to be valid in the light of subsequent structural determinations $[2,3]$. The suggestion that electron density from the hydrogen atoms of $\mathrm{BH}_{3}$ is back donated to form a $d \pi-p \pi$ bond between boron and phosphorus likewise fails to account for the difference between $\mathrm{PF}_{3}$ and $\mathrm{HPF}_{2}$ and, in fact, predicts a reverse effect.

To provide additional information which may contribute to understanding the factors involved, $\mathrm{HPF}_{2} \mathrm{BH}_{3}$ and several of its isotopic species have been studied by infrared and Raman spectroscopy. The assigned fundamentals have been subjected to a normal coordinate analysis using the compliance constant basis for comparison with earlier results on $\mathrm{PH}_{3} \mathrm{BH}_{3}$ and $\mathrm{PF}_{3} \mathrm{BH}_{3}$ [4]. 


\section{EXPERIMENTAL}

Difluorophosphine and the difluorophosphine-borane adducts were prepared as described in the literature $[1,5]$ and purified by trap-to-trap distillation on the vacuum line. Isotopically substituted varieties of diborane used to prepare the adducts were prepared by standard methods using $\mathrm{LiAlD}_{4}$ $(97 \% \mathrm{D})$ and enriched $\mathrm{BF}_{3}\left(99.4 \%{ }^{11} \mathrm{~B}\right.$ or $\left.92 \%{ }^{10} \mathrm{~B}\right) . \mathrm{DPF}_{2}$ was prepared via $\mathrm{PD}_{3}$ obtained from the reaction of calcium phosphide with $\mathrm{D}_{2} \mathrm{O}(99.6 \% \mathrm{D})$. All materials were handled in a standard vacuum line, particular care being paid to avoiding traces of adsorbed moisture. Purity of samples was verified by vapor pressure measurements, mass spectra and, when available, comparison with vibrational spectra in the literature. Since fluorophosphine compounds are quite sensitive to traces of air and moisture, all samples were freshly distilled before being subjected to spectroscopic examination, and cells and capillary tubes were pretreated before filling.

Infrared spectra of samples in the range from 200 to $4000 \mathrm{~cm}^{-1}$ were obtained with a Beckman IR-12 spectrometer. Gases were examined in a standard $10 \mathrm{~cm}$ cell with CsI windows while solids were studied in a low temperature cell similar to that described by Farran [6]. Raman spectra were recorded by a Spex 1401 Ramalog spectrometer using the $6328 \AA$ line of a $\mathrm{He}-\mathrm{Ne}$ laser and the $4880 \AA$ line of an $\mathrm{Ar}^{+}$laser as the exciting radiation. Samples were contained in sealed $1 \mathrm{~mm}$ capillaries which were positioned in the laser beam inside a vacuum jacketed tube. Cold dry nitrogen from liquid nitrogen boil-off was passed through the tube and the temperature monitored with a thermocouple. By adjusting the rate of flow of the nitrogen gas, the sample temperature could be maintained within about five degrees. The wavelength calibration of the infrared spectrometer was within about $0.2 \mathrm{~cm}^{-1}$ when checked against the spectra of the usual standard substances. The Raman spectrometer was calibrated with $\mathrm{Ar}^{+}$lines from the laser and checked against benzene. The uncertainty in band positions is estimated to be approximately $0.5 \mathrm{~cm}^{-1}$ for well defined bands.

\section{RESULTS AND ASSIGNMENTS}

Observed infrared and Raman frequencies for five isotopic species of $\mathrm{HPF}_{2} \mathrm{BH}_{3}$ in the solid, liquid and gaseous states are listed in Tables 1, 2 and 3. Infrared spectra for the normal and completely deuterated species in the gaseous and solid states are shown in Figs. 1 and 2 while the Raman spectrum of the hydrogen compound as a solid at $-150^{\circ} \mathrm{C}$ is displayed in Fig. 3 .

Compounds prepared from the naturally occurring mixture of boron isotopes are indicated by the symbol ${ }^{\mathrm{n}} \mathrm{B}$; compounds prepared from isotopically enriched boron are designated by the usual convention for isotopes.

As an eight atom molecule with $C_{\mathrm{s}}$ symmetry, $\mathrm{HPF}_{2} \mathrm{BH}_{3}$ has eighteen fundamental frequencies, eleven in the $A^{\prime}$ class and five in the $A^{\prime \prime}$. All frequencies are infrared and Raman active. To a good approximation, the 
TABLE 1

Observed infrared and Raman frequencies of $\mathrm{HPF}_{2}:{ }^{11} \mathrm{BH}_{3}$ and $\mathrm{HPF}_{2}:{ }^{10} \mathrm{BH}_{3}\left(\mathrm{~cm}^{-1}\right)$

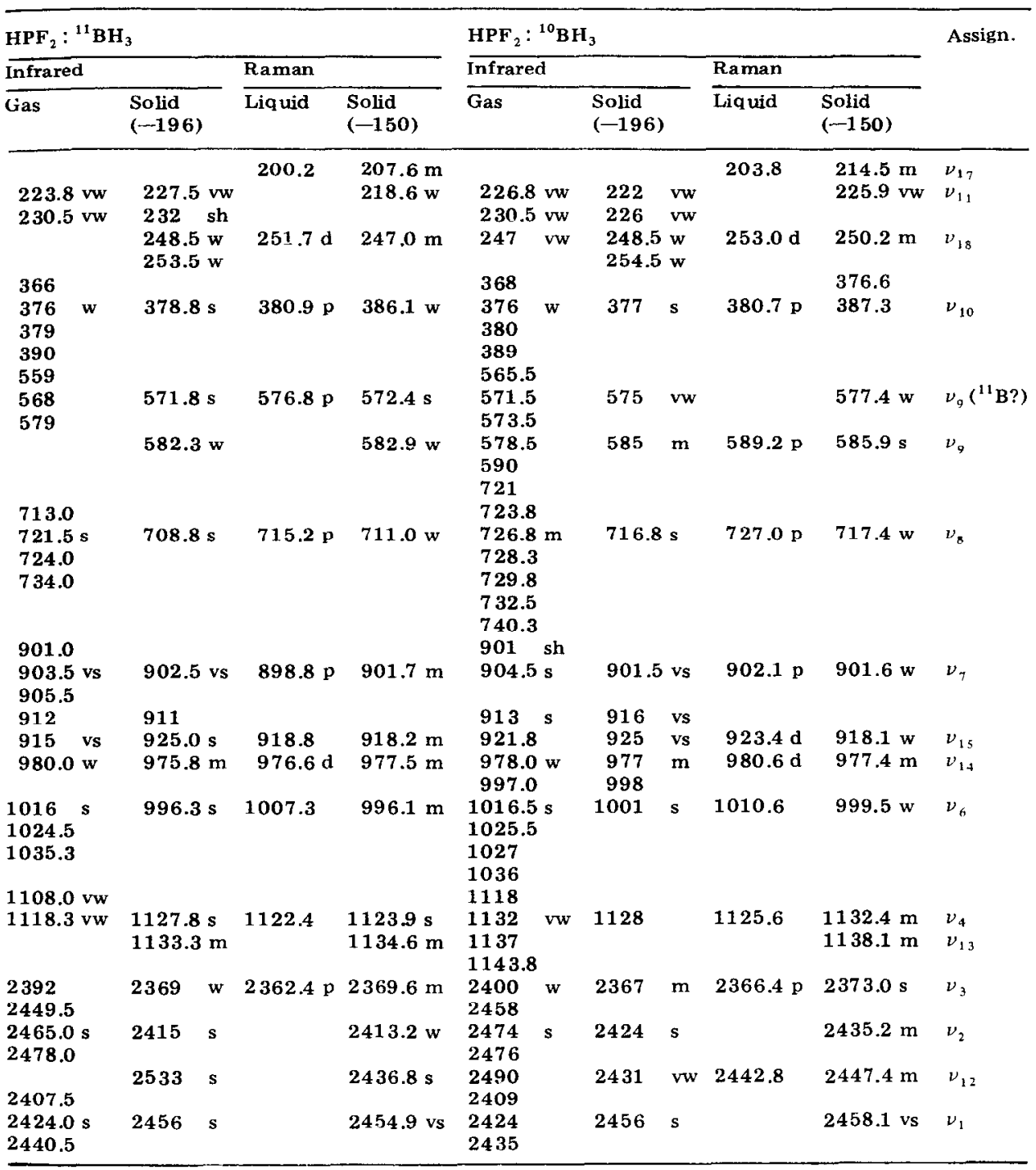

normal modes can be grouped into those primarily associated with the $\mathrm{HPF}_{2}$ part of the molecule, those local to the $\mathrm{BH}_{3}$ group, and those resulting from the formation of the $\mathrm{P}-\mathrm{B}$ dative bond. Table 4 gives the numbering scheme for the fundamentals together with a qualitative description of each mode. The frequencies associated with the $\mathrm{HPF}_{2}$ group were relatively easy to identify by comparison with the spectra of the free base [7]. Similarly, the characteristic $\mathrm{BH}_{3}$ group frequencies are well known [4, 8] although the 
TABLE 2

Observed infrared and Raman frequencies of $\mathrm{DPF}_{2}:{ }^{1 !} \mathrm{BH}_{3}$ and $\mathrm{HPF}_{2}:{ }^{11} \mathrm{BD}_{3}\left(\mathrm{~cm}^{-1}\right)$

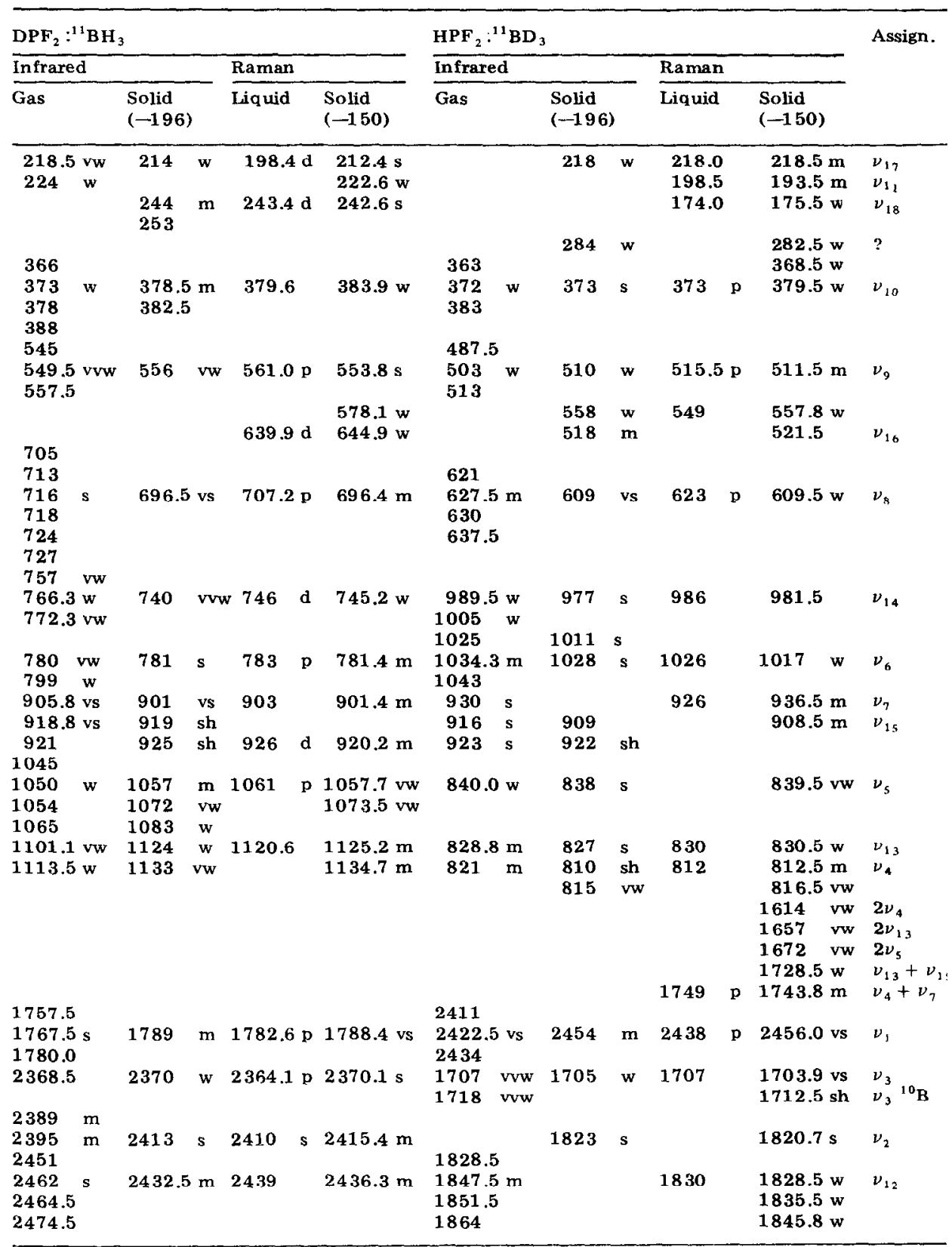


TABLE 3

Observed infrared and Raman frequencies of $\mathrm{DPF}_{2}:{ }^{11} \mathrm{BD}_{3}\left(\mathrm{~cm}^{-1}\right)$

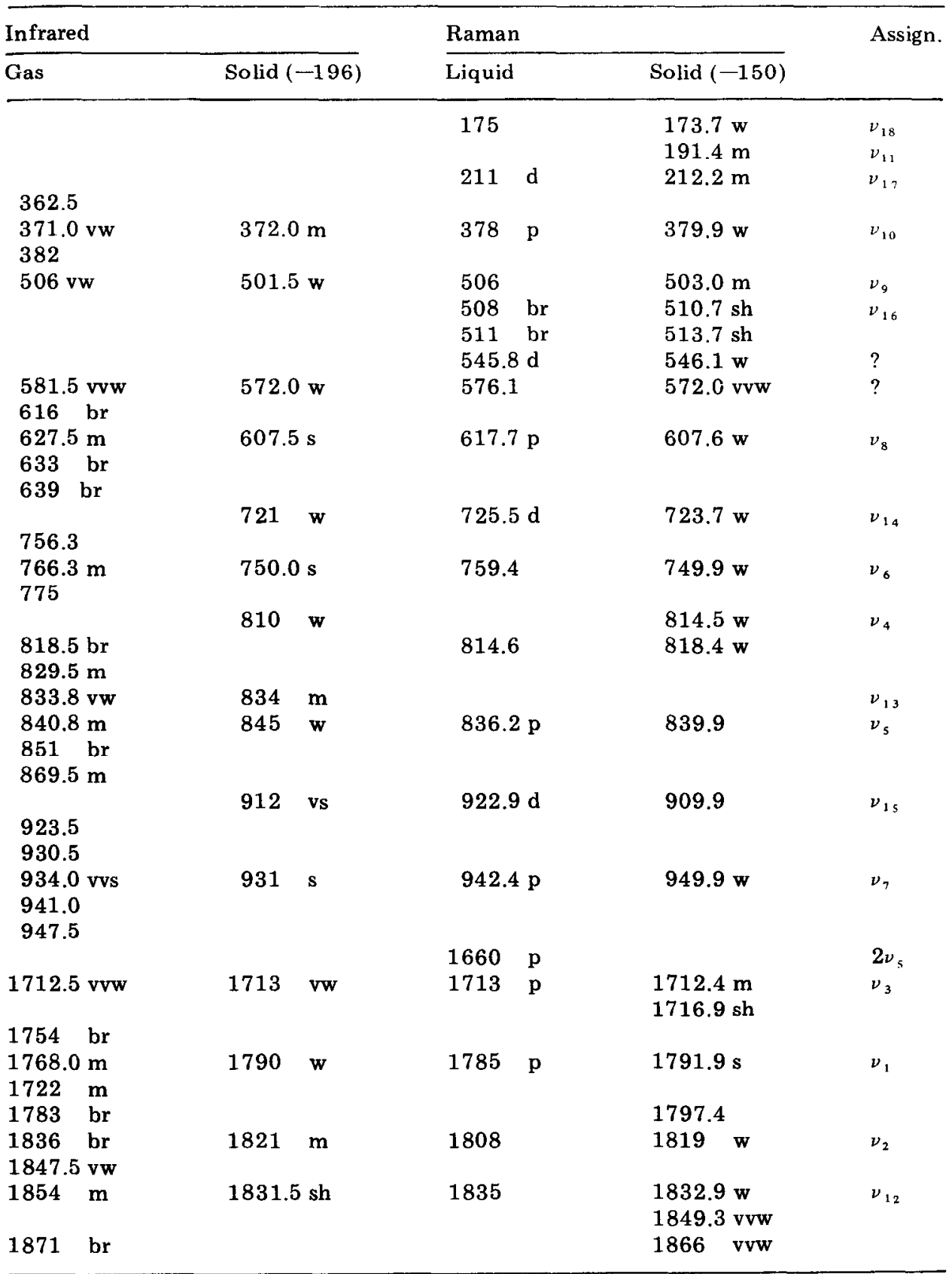

$\mathrm{w}=$ weak, $\mathrm{s}=$ strong, $\mathrm{br}=$ broad, $\mathrm{d}=$ depolarized, $\mathrm{p}=$ polarized, etc. 

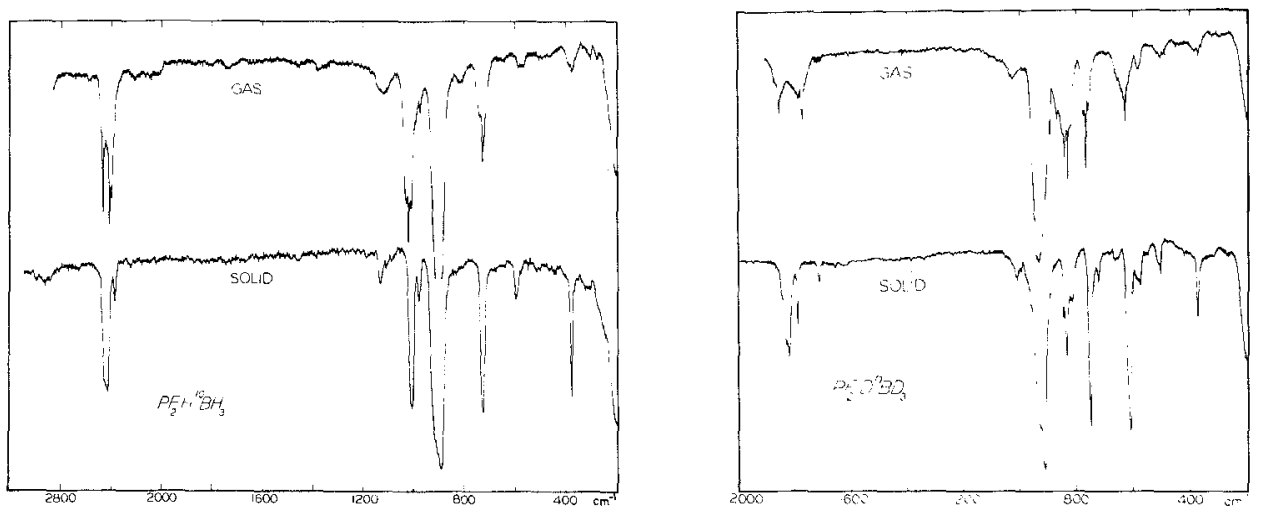

Fig. 1. Infrared spectra of difluorophosphine borane as a gas and as a solid at $-190^{\circ} \mathrm{C}$.

Fig. 2. Infrared spectra of deutero-difluorophosphine borane as a gas and as a solid at $-190^{\circ} \mathrm{C}$.

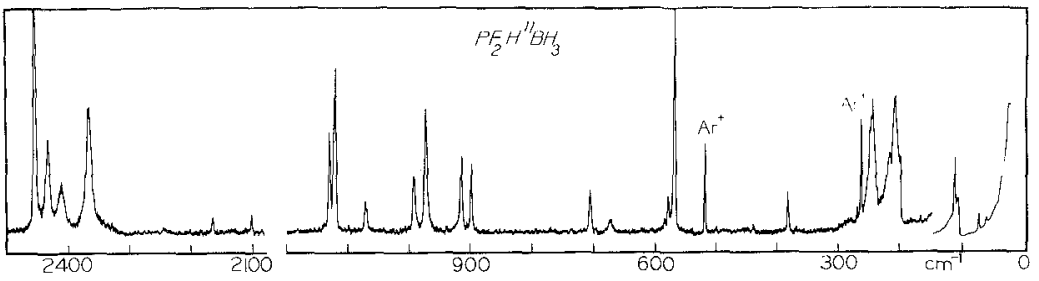

Fig. 3. Raman spectrum of solid $\mathrm{PF}_{2} \mathrm{HBH}_{3}$ at $-150^{\circ} \mathrm{C}$.

lower symmetry in the present case causes the degenerate modes to split. In the case of the $\mathrm{B}-\mathrm{H}$ stretching frequencies, this splitting amounted to about $40 \mathrm{~cm}^{-1}$. Since it was possible to deuterate selectively either end of the molecule, possible confusion between $\mathrm{P}-\mathrm{H}$ and $\mathrm{B}-\mathrm{H}$ modes could be eliminated. Proposed assignments accompany the experimental frequencies in Tables $1-3$, and the values for the eighteen fundamentals are summarized in Table 4. The discussion below focuses on selected frequencies for which assignment problems existed, or for which there are features of interest.

As in the case of $\mathrm{HPF}_{2}$, relatively strong intermolecular forces appear to exist in the condensed phases of the adduct. In particular, the $\mathrm{P}-\mathrm{H}$ and $\mathrm{P}-\mathrm{D}$ stretching bands shifted to higher frequency in the transition from gas to solid, the magnitude of the shift being some $30 \mathrm{~cm}^{-1}$ in the hydrogen species. Although the values are not quite as large as in the case of the free base [7], they do indicate significant interactions of a non-hydrogen bonding type involving the hydrogen on the phosphorus. The $\Lambda^{\prime \prime} \mathrm{BH}_{3}$ stretching mode $\left(v_{12}\right)$ also was sensitive to the gas solid transition but the shift was in the customary direction. Consequently, $\nu_{1}$ and $\nu_{12}$ interchanged relative positions in the spectrum of the solid compared to the gas spectrum. 
TABLE 4

Vibrational assignments for isotopic species of $\mathrm{HPF}_{2}: \mathrm{BH}_{3}$ and differences between observed and calculated frequencies $\left(\mathrm{cm}^{-1}\right)$

\begin{tabular}{|c|c|c|c|c|c|c|c|c|c|c|}
\hline \multirow[t]{2}{*}{ Description } & \multicolumn{2}{|c|}{$\mathrm{HPF}_{2}:{ }^{11} \mathbf{B H}_{3}$} & \multicolumn{2}{|c|}{$\mathrm{DPF}_{2}:{ }^{11} \mathrm{BH}_{3}$} & \multicolumn{2}{|c|}{$\mathrm{HPF}_{2}:{ }^{10} \mathrm{BH}_{3}$} & \multicolumn{2}{|c|}{$\mathrm{HPF}_{2}:{ }^{11} \mathrm{BD}_{3}$} & \multicolumn{2}{|c|}{$\mathrm{DPF}_{2}:{ }^{11} \mathrm{BD}_{3}$} \\
\hline & Freq. & $\Delta$ & Freq. & $\Delta$ & Freq. & $\Delta$ & Freq. & $\Delta$ & Freq. & $\Delta$ \\
\hline \multicolumn{11}{|l|}{$A^{\prime}$ Class } \\
\hline$\nu_{1} \quad \mathrm{P}-\mathrm{H}$ Stretch & 2455 & -14 & 1788 & 11 & 2458 & -11 & 2456 & -13 & 1792 & 15 \\
\hline$\nu_{2} \quad \mathrm{~B}-\mathrm{H}^{\prime}$ Stretch & 2413 & -4 & 2415 & -1 & 2435 & 3 & 1821 & 1 & 1819 & 1 \\
\hline$\nu_{3} \quad \mathrm{~B}-\mathrm{H}$ Stretch & 2370 & -1 & 2370 & -1 & 2373 & -2 & 1704 & -3 & 1712 & 5 \\
\hline$\nu_{4} \quad \mathbf{B H}_{2}$ Deform. & 1124 & -6 & 1135 & 12 & 1132 & -5 & 813 & 1 & 817 & -4 \\
\hline$\nu_{5} \quad \mathrm{BH}_{2}$ Deform. & 1076 & 5 & 1058 & -8 & 1086 & 4 & 840 & -3 & 840 & -6 \\
\hline$\nu_{6} \quad \mathrm{HPF}_{2}$ Deform. & 996 & -10 & 781 & -7 & 1000 & -10 & 1018 & 12 & 750 & -1 \\
\hline$\nu_{7} \quad \mathrm{P}-\mathrm{F}$ Stretch & 902 & -2 & 901 & -8 & 902 & -2 & 937 & 6 & 947 & 19 \\
\hline$\nu_{8} \quad \mathrm{BH}_{3}$ Wag & 711 & 3 & 696 & -5 & 717 & 0 & 610 & 0 & 607 & 4 \\
\hline$\nu_{9} \quad \mathrm{P}-\mathrm{B}$ Stretch & 572 & -12 & 554 & 2 & 586 & -6 & 512 & 4 & 503 & 8 \\
\hline$\nu_{10} \mathrm{PF}_{2}$ Deform. & 386 & -6 & 384 & -7 & 387 & -5 & 380 & 8 & 380 & 9 \\
\hline$\nu_{11} \quad \mathrm{HPF}_{2}$ Wag & 219 & -3 & 223 & 5 & 226 & 3 & 194 & -3 & 191 & -2 \\
\hline \multicolumn{11}{|l|}{$A^{\prime \prime}$ Class } \\
\hline$\nu_{12} \quad \mathrm{~B}-\mathrm{H}$ Stretch & 2437 & 4 & 2436 & 3 & 2447 & -2 & 1836 & 0 & 1833 & -3 \\
\hline$\nu_{13} \mathrm{H}^{\prime} \mathrm{BH}$ Deform. & 1135 & -3 & 1125 & -3 & 1138 & -5 & 831 & 8 & 831 & -2 \\
\hline$\nu_{14}$ HPF Rock & 978 & 1 & 745 & -16 & 977 & -2 & 982 & -1 & 724 & 8 \\
\hline$\nu_{15} \quad \mathrm{P}-\mathrm{F}$ Stretch & 918 & 4 & 920 & 1 & 918 & 4 & 909 & -3 & 910 & -4 \\
\hline$\nu_{16} \mathrm{BH}_{2}$ Rock & 677 & -15 & 645 & -12 & 687 & -6 & 522 & 13 & 512 & 9 \\
\hline$\nu_{17} \mathrm{PF}_{2}$ Rock & 208 & -5 & 212 & -1 & 215 & 0 & 219 & 5 & 212 & -1 \\
\hline$\nu_{18} \quad \mathrm{P}-\mathrm{B}$ Torsion & 247 & -2 & 243 & -2 & 250 & 0 & 176 & 4 & 174 & 2 \\
\hline
\end{tabular}

$\Delta=$ Observed - calculated.

The two $\mathrm{P}-\mathrm{F}$ stretching bands were easily identified by virtue of their infrared intensity. However, deuteration of the $\mathrm{BH}_{3}$ group resulted in a shift of the $A^{\prime}$ band to a higher frequency, opposite to the expected direction. This shift is understandable if one notes that the two $A^{\prime} \mathrm{BH}_{3}$ deformation modes are $150-200 \mathrm{~cm}^{-1}$ higher than the $\mathrm{P}-\mathrm{F}$ stretch in the hydrogen compound but shift to about $100 \mathrm{~cm}^{-1}$ lower in the deuterated. The repelling interaction between levels of the same symmetry apparently is sufficient to overcome the normal mass effect and cause the shift in the opposite direction. A secondary consequence of this interaction is the interchange of the relative positions of the $A^{\prime}$ and $A^{\prime \prime} \mathrm{P}-\mathrm{F}$ stretching modes between the hydrogen and deuterium species. Experimentally, the interchange was established by the observation that, although the two $\mathrm{P}-\mathrm{F}$ stretches were not well resolved in the Raman spectrum of the liquid, the low-frequency side of the composite band was clearly polarized in the spectrum of the hydrogen compounds while the high-frequency side was polarized in the spectra of the $\mathrm{BD}_{3}$ adducts. A similar interchange of the relative position of the $\mathrm{P}-\mathrm{F}$ stretches has been observed in the case of 
$\mathrm{PF}_{3} \mathrm{BH}_{3}$ [4]. It is perhaps worth mentioning that the potential energy distribution from the normal coordinate analysis shows the band assigned to the $A^{\prime} \mathrm{P}-\mathrm{F}$ stretch in the $\mathrm{BD}_{3}$ adducts to be much more strongly mixed than are any of the bands assigned to $\mathrm{P}-\mathrm{F}$ motions in the other isotopic species. This, again, is in accord with the explanation proposed above. Energy level interaction can also be invoked in the case of the $A^{\prime} \mathrm{P}-\mathrm{H}$ deformation $\left(\nu_{6}\right)$ which shifts upwards from $996 \mathrm{~cm}^{-1}$ in the $\mathrm{BH}_{3}$ compound to $1018 \mathrm{~cm}^{-1}$ in the $\mathrm{BD}_{3}$. Here, an $A^{\prime} \mathrm{BH}_{3}$ deformation mode lies only $80 \mathrm{~cm}^{-1}$ above the $\mathrm{P}-\mathrm{H}$ deformation in $\mathrm{HPF}_{2} \mathrm{BH}_{3}$ but the nearest $\mathrm{BD}_{3}$ deformation is separated nearly $180 \mathrm{~cm}^{-1}$ away on the low-frequency side.

$\Lambda$ nother fundamental which gave problems in assignment was the out-ofplane $\mathrm{BD}_{3}$ rocking mode, $\nu_{16}$. Initially this mode was assigned to weak but distinct bands in the vicinity of $545 \mathrm{~cm}^{-1}$ in the spectra of the two $\mathrm{BD}_{3}$ compounds. However, the normal coordinate calculations clearly indicated that this was not a good choice, the calculated values persistently falling in the range $500-510 \mathrm{~cm}^{-1}$. Closer inspection of the Raman spectra showed that the strong polarized bands assigned to the $\mathrm{P}-\mathrm{B}$ stretch were both accompanied by a weak shoulder on the high frequency side which did not change significantly in intensity between the two polarization spectra. Assignment of these shoulders to $\nu_{16}$ resulted in much better agreement in the normal coordinate calculations and improved product rule ratios. The weak bands originally selected are attributed to impurities, most likely hydrogen species.

The P-B stretch is of considerable interest in the spectra of Lewis complexes. As in most previous studies, it exhibited strong intensity in the Raman spectra but was quite weak in the vapor infrared spectra. However, there was no difficulty in identification, its intensity, polarization characteristics and location providing unequivocal evidence.

Assignments of all bands were confirmed using the normal coordinate calculations described in the next section. Comparison of isotope product rule ratios based on the assignments of Table 4 with theoretical ratios is made in Table 5. In general, the agreement is satisfactory although the percentage differences for a few ratios are some what larger than those usually attributed to anharmonicity effects. Considering the number of frequencies in each class and the high likelihood of small perturbations due to Fermi resonances, however, the data of Table 5 support the essential correctness of the proposed assignments. The isotopic species $\mathrm{HPF}_{2}{ }^{10} \mathrm{BH}_{3}$ was used for reference in the isotope rule ratios rather than the ${ }^{11} \mathrm{~B}$ species since data for the latter were obtained from the compounds with the natural isotope ratio and as a result, the frequency values are slightly less reliable than those for the ${ }^{10} \mathrm{~B}$ species.

\section{NORMAL COORDINATE CALCULATIONS}

The normal coordinate analysis of the assigned vibrational frequencies was carried out in terms of symmetry compliance constants to facilitate compari- 
TABLE 5

Product rule ratios for various isotopic species of $\mathrm{HPF}_{2}: \mathrm{BH}_{3}$

\begin{tabular}{|c|c|c|c|c|c|c|}
\hline \multirow[t]{2}{*}{ Ratio } & \multicolumn{3}{|l|}{$A^{\prime}$ Class } & \multicolumn{3}{|c|}{$A^{\prime \prime}$ Class } \\
\hline & Theor. & Expt. & Dev. $(\%)$ & Theor. & Expt. & Dev. $(\%)$ \\
\hline$\frac{\mathrm{HPF}_{2}{ }^{10} \mathrm{BH}_{3}}{\mathrm{IIPF}_{2}{ }^{11} \mathrm{BH}_{3}}$ & 1.066 & 1.104 & -3.8 & 1.030 & 1.068 & -3.6 \\
\hline$\frac{\mathrm{HPF}_{2}{ }^{10} \mathrm{BH}_{3}}{\mathrm{DPF}_{2}{ }^{11} \mathrm{BH}_{3}}$ & 2.067 & 2.027 & 1.9 & 1.418 & 1.478 & -0.4 \\
\hline$\frac{\mathrm{HPF}_{2}{ }^{10} \mathrm{BH}_{3}}{\mathrm{HPF}_{2}{ }^{11} \mathrm{BH}_{3}}$ & 5.323 & 5.064 & 4.8 & 3.674 & 3.366 & 8.4 \\
\hline$\frac{\mathrm{HPF}_{2}{ }^{10} \mathrm{BH}_{3}}{\mathrm{DPF}_{2}{ }^{11} \mathrm{BH}_{3}}$ & 10.370 & 9.601 & 7.4 & 5.061 & 4.866 & 3.9 \\
\hline
\end{tabular}

son with previous work on related molecules $[4,7]$. Although the advantages of the compliance constant basis for normal coordinate analyses have been cited by several authors $[9,10]$, this approach has seen limited application to date and additional treatments are much to be desired.

Details of the computational procedures have been described previously [4] and will not be repeated here. Figure 4 displays the atom numbering

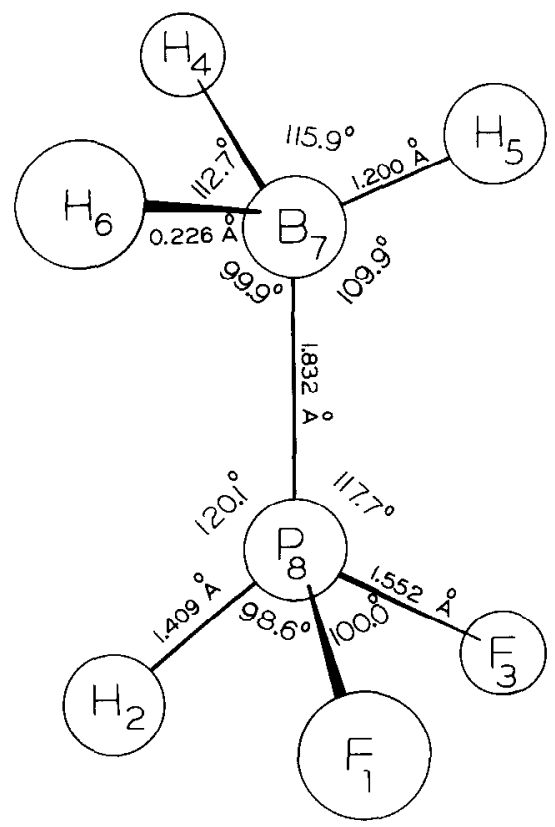

Fig. 4. Geometrical parameters and atom numbering scheme for difluorophosphine borane. 
TABLE 6

Symmetry coord inates for $\mathrm{PF}_{2} \mathrm{H} \cdot \mathrm{BH}_{3}$

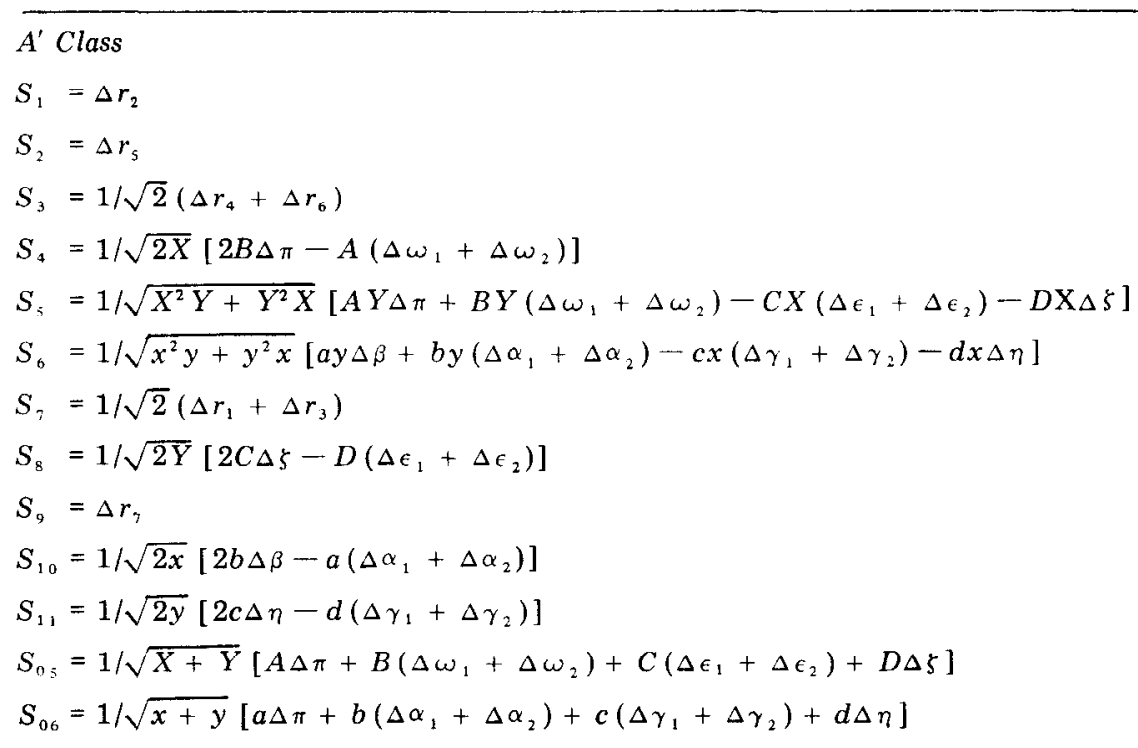

\section{$A^{\prime \prime}$ Class}

$S_{12}=1 / \sqrt{2}\left(\Delta r_{4}-\Delta r_{6}\right)$

$S_{13}=1 / \sqrt{2}\left(\Delta \omega_{1}-\Delta \omega_{2}\right)$

$S_{14}=1 / \sqrt{2}\left(\Delta \alpha_{1}-\Delta \alpha_{2}\right)$

$S_{1 \mathrm{~s}}=1 / \sqrt{2}\left(\Delta r_{1}-\Delta r_{3}\right)$

$S_{16}=1 / \sqrt{2}\left(\Delta \epsilon_{1}-\Delta \epsilon_{2}\right)$

$S_{17}=1 / \sqrt{2}\left(\Delta \gamma_{1}-\Delta \gamma_{2}\right)$

$S_{18}=: \Delta \tau$

$a=\cos \beta / 2 \sin \beta / 2$

$b=1 / 2 \alpha d \beta / d \alpha=\sin \alpha(\cos \alpha-\cos \gamma \cos \eta) / \sin ^{2} \eta$

$c=1 / 2 a d \beta / d \gamma=\sin \gamma(\cos \gamma-\cos \alpha \cos \eta) / \sin ^{2} \eta$

$d=a d \beta / d \eta=-2\left[\cos \alpha \cos \gamma \operatorname{cosec} \eta\left(2 \operatorname{cosec}^{2} \eta-1\right)-\cot \eta \operatorname{cosec}^{2} \eta\left(\cos ^{2} \gamma+\cos ^{2} \alpha\right)\right]$

$x=a^{2}+2 b^{2}$

$y=2 c^{2}+d^{2}$

$A=\cos \pi / 2 \sin \pi / 2$

$B=1 / 2 A d \pi / d \omega=\sin \omega(\cos \omega-\cos \epsilon \cos \xi) / \sin ^{2} \xi$

$C=1 / 2 A d \pi / d \epsilon=\sin \epsilon(\cos \epsilon-\cos \omega \cos \zeta) / \sin ^{2} \xi$

$D=A d \pi / d \zeta=-2\left[\cos \omega \cos \epsilon \operatorname{cosec} \zeta\left(2 \operatorname{cosec}^{2} \zeta-1\right)-\cot \zeta \operatorname{cosec}^{2} \zeta\left(\cos ^{2} \epsilon+\cos ^{2} \omega\right)\right]$

$X=A^{2}+2 B^{2}$

$Y=2 C^{2}+D^{2}$ 
TABLE 6 (continued)

Internal coordinate definitions

\begin{tabular}{|c|c|c|c|c|c|}
\hline Coordinate & Description & Def. & Coordinate & Description & Def. \\
\hline$r_{1}$ & $P-F$ & $1-8$ & $\pi$ & $\angle \mathrm{H}-\mathrm{B}-\mathrm{H}$ & $4-7-6$ \\
\hline$r_{2}$ & $\mathbf{P}-\mathbf{H}$ & $2-8$ & $\omega_{1}$ & $\angle \mathrm{H}-\mathrm{B}-\mathrm{H}^{\prime}$ & $4-7-5$ \\
\hline$r_{4}$ & B-H & $4-7$ & $\gamma_{1}$ & $\angle \mathbf{B}-\mathbf{P}-\mathbf{F}$ & $7-8-3$ \\
\hline$r_{5}$ & $\mathrm{~B}-\mathrm{H}^{\prime}$ & $5-7$ & $\eta$ & $\angle \mathrm{B}-\mathrm{P}-\mathrm{H}$ & $7-8-2$ \\
\hline$r_{7}$ & $\mathbf{P}-\mathbf{B}$ & $7-8$ & $\epsilon_{1}$ & $\angle \mathrm{P}-\mathrm{B}-\mathrm{H}$ & $8-7-4$ \\
\hline$\beta$ & $\angle \mathrm{F}-\mathrm{P}-\mathrm{F}$ & $1-8-3$ & $\xi$ & $\angle \mathrm{P}-\mathrm{B}-\mathrm{H}^{\prime}$ & $8-7-5$ \\
\hline$\alpha_{1}$ & $\angle \mathbf{H}-\mathbf{P}-\mathbf{F}$ & $2-8-3$ & $\tau$ & $\angle \mathrm{H}-\mathrm{P}-\mathrm{B}-\mathrm{H}^{\prime}$ & $2-8-7-5$ \\
\hline
\end{tabular}

Remaining coordinates defined by symmetry.

scheme and the structural parameters taken from the microwave work [3]. Table 6 lists the symmetry coordinates and the redundancy conditions around the phosphorus and boron atoms, and defines the internal coordinates in terms of atom numbers. The non-tetrahedral values for the angles around $B$ and $P$ atoms together with the low symmetry of the molecule led to rather complex expressions for the redundancy conditions and for the orthogonal $A^{\prime}$ symmetry coordinates involving angular coordinates. G matrix elements were computed using the expressions of Decius [11].

Initially, most of the off-diagonal compliance constants connecting nonadjacent coordinates were constrained to zero and removed from the fitting process. The sensitivities and the dispersions of the remaining offdiagonal constants were then examined with the result that several additional off-diagonal constants were also constrained to zero. The final fit utilized 22 constants for the $A^{\prime}$ class and 13 for the $A^{\prime \prime}$ and gave average deviations of 5.7 and $4.5 \mathrm{~cm}^{-1}$, respectively. Table 4 shows the individual differences between observed and calculated frequencies. The final symmetry compliance constants are given in Table 7 together with their dispersions. Inversion of the compliance matrices provided values for the corresponding symmetry force constants. In contrast to the compliance matrices, the force constant matrices contained very few zero off-diagonal elements. However, many of these entries were relatively small and have not been listed in Table 7. Potential energy distributions have not been tabulated here but showed no unexpected features. With very few exceptions, the principal contribution to the potential energy associated with each frequency resided in the assigned symmetry coordinate. The $\mathrm{P}-\mathrm{B}$ stretch in the hydrogen compounds could be rather clearly identified with a frequency in the range $570-585 \mathrm{~cm}^{-1}$. The presence of deuterium in the molecule, however, caused the $\mathrm{P}-\mathrm{B}$ stretch to mix extensively with the symmetric $\mathrm{BH}_{3}$ rocking mode and its identification then became ambiguous. 
TABLE 7

Symmetry compliance and force constants for $\mathrm{HPF}_{2} \mathrm{BII}_{3}$

\begin{tabular}{|c|c|c|c|c|c|c|c|}
\hline \multirow[t]{2}{*}{ Index } & \multicolumn{2}{|c|}{ Compliance } & \multirow{2}{*}{$\begin{array}{l}\text { Force } \\
\text { const. }\end{array}$} & \multirow[t]{2}{*}{ Index } & \multicolumn{2}{|c|}{ Compliance } & \multirow{2}{*}{$\begin{array}{l}\text { Force } \\
\text { const. }\end{array}$} \\
\hline & Const. & Disp. $^{a}$ & & & Const. & Disp. & \\
\hline \multicolumn{8}{|c|}{$A^{\prime}$ Class } \\
\hline 1,1 & 0.285 & 0.003 & 3.503 & 2,3 & -0.004 & 0.016 & 0.040 \\
\hline 2,2 & 0.403 & 0.072 & 2.932 & 2,5 & 0.336 & 0.130 & -0.538 \\
\hline 3,3 & 0.330 & 0.045 & 3.191 & 2,6 & - & - & 0.101 \\
\hline 4,4 & 2.740 & 0.056 & 0.385 & 2,7 & - & - & 0.065 \\
\hline 5,5 & 1.901 & 0.137 & 0.644 & 2,9 & - & - & 0.110 \\
\hline 6,6 & 1.510 & 0.251 & 0.742 & 3,4 & -0.216 & 0.233 & 0.251 \\
\hline 7,7 & 0.179 & 0.004 & 5.887 & 4,6 & -0.043 & 0.078 & 0.013 \\
\hline 8,8 & 5.240 & 0.525 & 0.299 & 5,6 & 0.262 & 0.118 & -0.121 \\
\hline 9,9 & 0.394 & 0.025 & 2.566 & 5,7 & - & - & -0.078 \\
\hline 10,10 & 0.752 & 0.117 & 1.431 & 5,9 & 0.081 & 0.040 & -0.131 \\
\hline \multirow[t]{3}{*}{11,11} & 3.185 & 0.171 & 0.505 & 6,7 & -0.116 & 0.029 & 0.479 \\
\hline & & & & 6,10 & -0.178 & 0.053 & 0.182 \\
\hline & & & & 7,10 & - & - & 0.118 \\
\hline 9,10 & 0.007 & 0.023 & -0.019 & 8,10 & - & - & -0.081 \\
\hline 10,11 & 0.252 & 0.191 & -0.174 & 8,11 & -2.419 & 0.152 & 0.233 \\
\hline \multicolumn{8}{|c|}{$A^{\prime \prime}$ Class } \\
\hline 12,12 & 0.329 & 0.024 & 3.061 & 12,13 & 0.079 & 0.183 & -0.079 \\
\hline 13,13 & 3.599 & 0.588 & 0.326 & 13,14 & 0.401 & 0.366 & -0.073 \\
\hline 14,14 & 1.948 & 0.411 & 0.571 & 13,16 & -1.014 & 0.359 & 0.137 \\
\hline 15,15 & 0.191 & 0.004 & 5.443 & 14,15 & 0.114 & 0.028 & -0.341 \\
\hline 16,16 & 2.511 & 0.080 & 0.470 & 14,17 & -0.428 & 0.052 & 0.106 \\
\hline 17,17 & 2.314 & 0.043 & 0.452 & 15,17 & - & - & -0.063 \\
\hline 18,18 & 4.114 & 1.274 & 0.253 & 16,18 & 0.608 & 0.339 & -0.069 \\
\hline
\end{tabular}

${ }^{\text {a }}$ Dispersions.

\section{DISCUSSION}

In order to compare data from the present work with those of other molecules of interest, valence potential constants rather than symmetry constants are desirable. These can be calculated straightforwardly in the case of bond stretches using equations in Table 6 defining the symmetry coordinates. The principal force and compliance constants of interest are given in Table 8 together with corresponding literature values for $\mathrm{HPF}_{2}$ and the borane complexes of $\mathrm{PH}_{3}$ and $\mathrm{PF}_{3}[4,7]$. Inspection of the table shows that the compliance constants for the $\mathrm{P}-\mathrm{F}$ and $\mathrm{P}-\mathrm{B}$ bonds in $\mathrm{PF}_{3} \mathrm{BH}_{3}$ and $\mathrm{HPF}_{2} \mathrm{BH}_{3}$ are virtually identical and the same can be said for the $\mathrm{B}-\mathrm{H}$ bonds with the exception of the single in-plane $\mathrm{B}-\mathrm{H}$ bond in $\mathrm{HPF}_{2} \mathrm{BH}_{3}$. On the other hand, the $\mathrm{P}-\mathrm{B}$ bond in $\mathrm{PH}_{3} \mathrm{BH}_{3}$ is clearly weaker (more compliant) than it is in the other two molecules, which is consonant with the fact that $\mathrm{PH}_{3} \mathrm{BH}_{3}$ is the weakest of the three complexes. 
TABLE 8

Valence stretching force and compliance constants for various phosphine boranes ( $u$ nits = md/Ångström)

\begin{tabular}{llll}
\hline & $\mathrm{HPF}_{2} \mathrm{BH}_{3}{ }^{\mathrm{a}}$ & $\mathrm{PF}_{3} \mathrm{BH}_{3}{ }^{\mathrm{b}}$ & $\mathrm{PH}_{3} \mathrm{BH}_{3}{ }^{\mathrm{b}}$ \\
\hline Force constants & 5.665 & & \\
P-F & 3.503 & 5.40 & 3.39 \\
P-H & 2.932 & - & 3.04 \\
B-H (ip) & 3.126 & 3.22 & 1.95 \\
B-H (op) & 2.566 & 2.56 & \\
P-B & 0.222 & & \\
PF, PF & & & 0.295 \\
Compliance constants & 0.185 & 0.186 & 0.344 \\
P-F & 0.285 & - & 0.525 \\
P-H & 0.403 & 0.313 & \\
B-H (ip) & 0.330 & 0.405 & \\
B-H (op) & 0.394 & & \\
P-B & -0.006 & & \\
PF, PF & & & \\
\hline
\end{tabular}

${ }^{a}$ This work. b Reference 4.

The marked similarity found in the bond compliance constants of $\mathrm{HPF}_{2} \mathrm{BH}_{3}$ and $\mathrm{PF}_{3} \mathrm{BH}_{3}$ provides no insight regarding the significant difference in stability of the two complexes. However, some comments can be made. Staplin and Parry [12] regard the unusual stability of $\mathrm{HPF}_{2} \mathrm{BH}_{3}$ as being due to a specific type of interaction between the protonic hydrogen on the $\mathrm{HPF}_{2}$ group and the hydridic hydrogens of the $\mathrm{BH}_{3}$. The basis of this suggestion is that the microwave data [3] show that the $\mathrm{BH}_{3}$ group is tilted towards the hydrogen on the phosphorus and away from the fluorines. However, the present calculations show that the compliance constant of the $\mathrm{P}-\mathrm{H}$ bond in $\mathrm{HPF}_{2} \mathrm{BH}_{3}$ does not differ significantly from that of the $\mathrm{P}-\mathrm{H}$ bond in $\mathrm{PH}_{3} \mathrm{BH}_{3}$ and, moreover, is significantly less compliant than it is in free $\mathrm{HPF}_{2}$ [7]. This type of behavior is not characteristic of a proton involved in hydrogen bonding through electrostatic interaction and suggests that the $\mathrm{H}^{+} \cdots \mathrm{H}^{-}$intramolecular attraction may not be as important as thought. Support for this conclusion can be found in ab initio calculations recently carried out by Armstrong [13] which lead to the conclusion that the interaction of the hydrogen on the phosphorus with the $\mathrm{BH}_{3}$ group, while significant, is only one of three effects energetically all about the same magnitude. His view is that the unexpected stability of the $\mathrm{HPF}_{2}$ borane complex is due to the cumulative effect of these effects which are not present, or are small, in the $\mathrm{PF}_{3}$ complex. Although Armstrong did not calculate force or compliance constants in his work, he gave overlap populations for all bonds. If these are considered a measure of relative bond strengths, his comparison between $\mathrm{HPF}_{2} \mathrm{BH}_{3}$ and $\mathrm{PF}_{3} \mathrm{BH}_{3}$ is quite similar to the comparison based on 
compliance constants found here. The only disagreement is that he finds a higher overlap population, and presumably a stronger bond, for the unique in-plane $\mathrm{B}-\mathrm{H}$ bond of the $\mathrm{HPF}_{2}$ complex whereas the normal coordinate calculations show this bond to be weaker than the other two $\mathrm{B}-\mathrm{H}$ bonds.

\section{ACKNOWLEDGEMENT}

This work was supported in part by the National Science Foundation under Grant GP-8249. This assistance is gratefully acknowledged.

\section{REFERENCES}

1 R. W. Rudolph and R. W. Parry, J. Am. Chem. Soc., 89 (1967) 1621.

2 R. L. Kuczkowski, J. Am. Chem. Soc., 90 (1968) 1705.

3 J. P. Pasinski and R. L. Kuczkowski, J. Chem. Phys., 54 (1971) 1903.

4 R. C. Taylor, R. W. Rudolph, R. J. Wyma and V. D. Dunning, J. Raman Spectrosc., 2 (1974) 175.

5 R. W. Rudolph and R. W. Parry, Inorg. Chem., 4 (1965) 1339.

6 C. F. Farran, Dissertation, The University of Michigan, 1966.

7 V. D. Dunning and R. C. Taylor, Spectrochim. Acta, Part A, 35 (1979) 479.

8 L. H. Jones, R. C. Taylor and R. T. Paine, J. Chem. Phys., 70 (1979) 749.

9 J. C. Decius, J. Chem. Phys., 38 (1963) 241.

10 L. H. Jones and R. R. Ryan, J. Chem. Phys., 52 (1970) 2003.

11 J. C. Decius, J. Chem. Phys., 17 (1949) 1217.

12 D. C. Staplin and R. W. Parry, Inorg. Chem., 18 (1979) 1473.

13 D. R. Armstrong, Inorg. Chim. Acta, 13 (1975) 121. 